\title{
Melhora dos índices de aprovação pela formação de turmas com perfis de conhecimento semelhantes
}

\author{
Robinson Vida Noronha , Hilton José Silva de Azevedo \\ Universidade Tecnológica Federal do Paraná (UTFPR) \\ Departamento Acadêmico de Eletrônica \\ Curitiba - Paraná - Brazil \\ \{vida, hilton\}@utfpr.edu.br
}

\begin{abstract}
Resumo. Investigou-se se a formação de turmas de acordo com o coeficiente de rendimento do aluno poderia ter um desempenho acadêmico superior às turmas que não possuem esse tipo de critério de composição. Todas as turmas envolvidas nesse estudo tiveram os mesmos instrumentos de avaliação. As turmas formadas por alunos de baixo coeficiente de rendimento tiveram uma abordagem didática diferenciada. Os resultados obtidos apontam para um aumento nos índices de aprovação e reprovação das turmas e uma redução nos indices de desistência dos alunos.
\end{abstract}

\begin{abstract}
This paper report research results of the impact of clustering students by student's score criterion to student's success, failure and abandon of a particular class. The students with low student's score received a particular didactic strategy. The results of this research indicate that when classes are formed by student's score criterion, the index of number of student's success and student's failure increase. The results also indicate a reduction of the index of number of student's abandon of the class. All students that participate of this research (322) was assessed with the same tests and project's defense.
\end{abstract}

\section{Introdução}

Ao ingressar em um curso superior em uma universidade, o aluno traz consigo a expectativa, da formatura. Porém, nem sempre isso ocorre. De acordo com [Gaioso 2006], [Manhães et al. 2012], [Costa et al. 2014], [Souza et al. 2015], entre outros, um dos problemas enfrentados pelas universidades é o crescente número da Evasão Escolar. Investigar as causas e possíveis estratégias que minimizem esse problema tem sido alvo de diversas investigações sem que o problema ainda tenha sido resolvido. Conforme apresentado na Seção 2 deste artigo, alguns relatos de pesquisa associam a Evasão Escolar ao elevado número de disciplinas reprovadas pelos alunos em um curso e à motivação de seus alunos.

Nesse contexto, Jesus (2008) destaca que os professores deveriam utilizar um ritmo de ensino adequado às capacidades e conhecimentos anteriores dos alunos como forma de motivá-los e em decorrência disso, reduzir os índices de reprovação. Porém, como fazer isso com as turmas heterogêneas de alunos? Nos dias de hoje, é comum o professor identificar nas disciplinas de programação de computadores, por exemplo, alguns alunos que já possuem conhecimento e habilidades de programação, enquanto que outros não as apresentam. 
A presença de pelo menos esses dois tipos de alunos na mesma turma faz com que essa turma seja um desafio ao professor e em alguns casos, uma possível fonte de desmotivo para alguns alunos. O conjunto de atividades, a forma de abordagem dos conteúdos deveriam ser adaptados por esse professor aos diversos tipos de alunos. Essa adaptação poderia ter o objetivo de melhorar o desempenho dos alunos e que eles tivessem a sensação de serem capaz de executar as tarefas solicitadas.

Este trabalho de pesquisa apresenta um estudo de caso que busca identificar alguns efeitos no desempenho acadêmico de alunos quando o critério de seleção dos alunos na turma é Coeficiente de Rendimento Acadêmico desses alunos. Define-se assim, duas turmas, uma composta por alunos com coeficiente de rendimento elevado e outra com alunos com coeficiente de rendimento acadêmico baixo. Investiga-se também se uma pequena mudança nas estratégias pedagógicas do professor é capaz de modificar o desempenho acadêmico dos alunos com coeficiente de rendimento baixo.

A disciplina escolhida para o experimento é a Fundamentos de Programação 2. Essa disciplina é ofertada no segundo período do curso de Engenharia Eletrônica da Universidade Tecnológica Federal do Paraná. Os alunos que cursam essa disciplina são divididos em duas turmas. $\mathrm{O}$ início do estudo foi no segundo semestre acadêmico de 2013 e continua em andamento. Participaram desse estudo quatro professores e 322 alunos no total. Os dados coletados são contrastados nessa pesquisa com uma terceira turma (turma referência ou controle) que não teve o mesmo critério de formação, o coeficiente de rendimento acadêmico dos alunos.

Para descrever o estudo de caso e seus resultados, este artigo está estruturado da seguinte maneira: a Seção 2 analisa alguns trabalhos a respeito de formação de grupo e desempenho e possíveis fatores associados à Evasão Escolar. Na Seção 3, o experimento é descrito. A análise dos dados coletados é feita na Seção 4 e uma discussão é apresentada na Seção 5. Conclui-se este artigo com trabalhos futuros na Seção 6.

\section{Estado da Arte}

Um dos motivos que poderia ser considerado como uma das causas da Evasão Escolar, é o baixo desempenho acadêmico do aluno [Manhães et al 2012], [Souza et al. 2015] e [Santos et al 2015]. Porém, outro ponto de vista desse fenômeno poderia indicar que tanto a Evasão Escolar como o baixo desempenho acadêmico do aluno estão relacionados com a falta de engajamento do aluno com o curso ou com a falta de motivação desse aluno. Independentemente do ponto de vista considerado, há de se constatar que existem indícios da existência de relacionamento entre a Evasão Escolar e o baixo desempenho acadêmico do aluno.

Ao estudar a evasão em um curso de Licenciatura em Informática do IFRN, o trabalho de [Souza et al 2015] identificou, por meio de entrevistas com esses alunos dois motivos destacados neste trabalho que indicam que o desempenho acadêmico individual do aluno pode estar associado à evasão escolar. Os motivos identificados pelos alunos como causas do abandono foram, entre outros: i) a dificuldade de acompanhar a disciplina de algoritmos e outras disciplinas técnicas e ii) os métodos de ensino, métodos de avaliação e dificuldade de relacionamento com os professores. 
Outro trabalho que também relaciona o abandono com a dificuldade encontrada pelos alunos em disciplinas é o de Manhães et al. (2012). Por meio de técnicas de mineração de dados, Manhães et al. identificaram em bases de dados computacionais uma redução gradual da quantidade de disciplinas aprovadas até o momento da evasão do curso dos alunos. Essa redução apontada por Manhães et al, bem como a pouca quantidade de empréstimos de livros na biblioteca feitas pelos alunos que desistem da universidade [Oliveira Junior et al. 2014], poderiam ser interpretadas como um indício da pouca motivação desses alunos em relação às disciplinas e ao curso. O efeito da motivação nos índices de aprovação em disciplinas de programação é constatado por Santos et al. (2015).

Além desses aspectos, [Barker et al, 2009] apontam que o relacionamento entre os alunos é um fator importante a ser considerado quando o assunto é Evasão Escolar, ou retenção de alunos. O trabalho de Barker et al, relata que as chances do aluno permanecer no curso aumentam quando ele se sente parte de uma comunidade de mesmo nível social e intelectual. O que é esperado que aconteça neste experimento quando as turmas são divididas pelo coeficiente de rendimento. Ou seja, que os alunos desistam menos da disciplina e, consequentemente, haja um aumento no número de aprovados ou reprovados.

Diversos estudos a respeito de formação de grupos tem apresentado resultados que, de acordo com [Citadin et al. 2014], não podem ser generalizados. Depende da tarefa que o grupo realizará. Por exemplo, de acordo com [Manukian et al, 2013] grupos homogêneos (grupos com as mesmas características) são melhores para disseminar conhecimento.

\section{O Experimento}

Investigou-se o impacto que duas abordagens: uma de gestão e outra didática teriam sobre o desempenho de estudantes de uma disciplina de programação de segundo semestre do primeiro ano de um curso de Engenharia Eletrônica. A abordagem de gestão consistiu em dividir parte dos estudantes em dois grupos, um com os maiores coeficientes de rendimento escolar e o outro com os menores e observar se essa divisão tinha impacto no desempenho acadêmico.

A abordagem didática consistiu em oferecer um tratamento diferenciado para um grupo de estudantes com menores coeficientes de rendimento. $\mathrm{O}$ estudo nasceu de uma iniciativa espontânea de um dos professores da disciplina que buscavam alternativas para reduzir a quantidade de estudantes repetentes nessa disciplina.

O método empregado foi o experimental investigativo. A pergunta de pesquisa procurou responder se o fato de se separar os alunos fortes dos fracos em uma turma poderia ter um impacto positivo no desempenho dos estudantes de uma disciplina de programação. Como o fato motivador era a busca de ações que pudessem contribuir para a redução da quantidade de alunos repetentes em turmas iniciais do curso de engenharia, decidiu-se que a divisão não seria o foco principal mas o que se poderia fazer para auxiliar os prováveis futuros repetentes. Isso provocou o desenho de uma abordagem diferenciada para o grupo dos estudantes mais fracos. Para assegurar o rigor do processo de ensino aprendizagem, todos os participantes foram submetidos aos mesmos instrumentos de avaliação. 


\subsection{Caracterização da disciplina.}

A disciplina Fundamentos de Programação 2, objeto deste estudo, é organizada em dois encontros de duas aulas semanais cada. Nessa disciplina, ofertada no segundo semestre do curso de engenharia eletrônica, os estudantes são apresentados ao paradigma Orientado a Objeto, tendo como plataforma a linguagem "C++". Os aprovados devem demonstrar compreender e empregar os conceitos de classe, herança, polimorfismo, abstração e habilidades no desenvolvimento e documentação de projetos de software, entre outros. A avaliação é feita mediante provas escritas e um projeto de desenvolvimento de "software" que deve ser defendido perante banca formada pelos professores da disciplina. $\mathrm{O}$ estudo cobriu cinco semestres consecutivos em que a disciplina foi ofertada em três turmas por vez.

\subsection{Organização das turmas.}

A distribuição dos estudantes nas turmas foi condicionada ao regulamento de matrícula vigente na universidade. Foram ofertados: um horário noturno com um terço das vagas disponíveis e um horário vespertino com dois terços das vagas disponíveis. O horário noturno serviria como grupo de referência (o preenchimento da turma teve seus coeficientes variados em função das demandas espontâneas dos estudantes que se matricularam e o professor conduziu a disciplina sem mudanças de abordagem em relação aos semestres anteriores ao experimento). O horário vespertino foi organizado em duas turmas, intencionalmente divididas em função dos maiores ou menores coeficientes de rendimento dos inscritos.

Devido a peculiaridades administrativas, o corpo docente participa de vários cursos (por exemplo: Engenharia Eletrônica, Engenharia de Computação, Engenharia Mecatrônica e Tecnologia de Telecomunicações) e nem sempre o mesmo professor pode assumir as mesmas disciplinas ao longo dos semestres. Esse fato fez com que quatro professores atuassem na disciplina de Fundamentos de Programação 2 ao longo dos cinco semestres do experimento.

\subsection{Caracterização da mudança de abordagem pedagógica.}

A alteração da abordagem consistiu na distribuição, para as turmas com menores coeficientes de rendimento, de tarefas semanais de resolução de exercícios em casa e entrega para o professor, correção dos exercícios pelo professor (extra classe) e posterior devolução dos mesmos e apresentação de exemplos de resolução dos exercícios em aula.

\subsection{Dados considerados no experimento.}

Os dados do experimento foram obtidos diretamente dos diários de classe: Média de Desempenho, situação do estudante ao final da disciplina (aprovado, reprovado, desistente). Todos os estudantes realizaram as mesmas avaliações. A nota relativa ao projeto no final do semestre, das três turmas de cada semestre, foi construída mediante defesa perante banca composta pelos quatro professores.

\subsection{Critérios para análise dos dados.}

Como somente um dos quatro professores mudou sua abordagem pedagógica quando trabalhava com as turmas com baixo coeficiente de rendimento, adotou-se o 
agrupamento apresentado na Tabela 1, para as 15 turmas que participaram do experimento (3 turmas a cada semestre entre 2013/2 e 2015/2).

As variáveis principais deste experimento são: número de aprovados, reprovados e desistentes nos três grupos. A variável secundária considerada é a média de rendimento nos grupos dos aprovados e reprovados.

Tabela 1 - Agrupamento das Turmas

\begin{tabular}{|l|l|}
\hline GrupoBN & Grupo Baixo coeficiente abordagem Não diferenciada (46 estudantes) \\
\hline GrupoBD & Grupo Baixo coeficiente abordagem Diferenciada (64 estudantes) \\
\hline GrupoCA & $\begin{array}{l}\text { Grupo Coeficiente ALTO e sem abordagem diferenciada (100 } \\
\text { estudantes) }\end{array}$ \\
\hline GrupoRE & $\begin{array}{l}\text { Grupo de referência. Coeficientes misturados e sem abordagem } \\
\text { diferenciada(112 estudantes). Os estudantes que participam dessa turma } \\
\text { são estudantes que já cursaram a disciplina pelo menos uma vez e } \\
\text { repetiram por nota ou nota e frequência. }\end{array}$ \\
\hline
\end{tabular}

\section{Análise dos dados obtidos}

Nessa seção apresenta-se os dados coletados a partir de consultas em um Sistema de Gerenciamento de Informações Acadêmicas. Esses dados estão agrupados e são apresentados nas seguintes seções:

* Seção 4.1: Dados de desempenho acadêmico entre os grupos com baixo coeficiente de rendimento acadêmico

* Seção 4.2: Dados de desempenho acadêmico do grupo com coeficiente de rendimento acadêmico elevado.

* Seção 4.3: Dados de desempenho acadêmico dos grupos com coeficiente de rendimento acadêmico alto e baixo com abordagem diferenciada e da turma com os coeficientes misturados (grupo controle).

* Seção 4.4: Dados de desempenho acadêmico total das turmas divididas por coeficiente acadêmico alto e baixo com e sem abordagem diferenciada e a turma com os coeficientes misturados (grupo controle).

\subsection{Dados relativos de desempenho entre os grupos com baixo coeficiente acadêmico.}

A Tabela 2 compara os resultados gerais de desempenho, entre os grupos com baixo coeficiente acadêmico, tendo como variável de controle a adoção ou não de abordagem diferenciada. Lembrando que as provas são as mesmas nos dois grupos, observou-se um desempenho levemente melhor no grupo de estudantes com baixo coeficiente que fez exercícios extras de fixação:

- Uma média de rendimento ligeiramente superior $(6,9$ contra 6,6$)$, 
- 5,5\% a mais de estudantes aprovados,

- $16 \%$ a menos de estudantes reprovados,

- um aumento de $10,5 \%$ no número de desistentes e

- apesar de bastante baixa, uma melhora na média de rendimento dos reprovados que passou de 1,9 para 2,8 (aumento de $80 \%$ ).

Tabela 2 Comparação de desempenho e frequências médios entre turmas com atividade específica para os mais fracos.

\begin{tabular}{|c|c|c|c|c|c|c|}
\hline & \multicolumn{3}{|c|}{$\begin{array}{l}\text { BAIXO COEF. ABORDAGEM PADRÃO - } \\
\text { GrupoBN }\end{array}$} & \multicolumn{3}{|c|}{$\begin{array}{l}\text { BAIXO COEF. ABORDAGEM DIFERENCIADA - } \\
\text { GrupoBD }\end{array}$} \\
\hline & $\%$ & $\begin{array}{l}\text { MÉDIA DA } \\
\text { TURMA }\end{array}$ & DESV. PADRAO & $\%$ & $\begin{array}{l}\text { MÉDIA DA } \\
\text { TURMA }\end{array}$ & DESV. PADRAO \\
\hline APROVADOS & $19,57 \%$ & 6,6 & 0,5 & $25,00 \%$ & 6,9 & 0,9 \\
\hline REPROVADOS & $63,04 \%$ & 1,9 & 1,2 & $46,88 \%$ & 2,8 & 1,7 \\
\hline DESISTENTES & $17,39 \%$ & ---- & ---- & $28,13 \%$ & --- & --- \\
\hline
\end{tabular}

\subsection{Dados Referentes ao grupo de coeficiente alto}

A Tabela 3 compara os resultados gerais de desempenho acadêmico médio, entre os grupos Referência (GrupoRE) e Coeficiente Alto - GrupoCA. Observou-se algumas melhoras nos índices do GrupoCA, quando comparado ao Grupo RE, a saber:

- A quantidade de aprovados foi maior - $74 \%$ para o GrupoCA e $43 \%$ para o GrupoRE,

- A quantidade de reprovados foi menor - $16 \%$ para o GrupoCA e $27 \%$ para o GrupoRE,

- A quantidade de desistentes foi menor - 10\% para o GrupoCA e 30\% para o GrupoRE,

- A média das turmas permaneceu praticamente a mesma $(7,8)$ nos grupos GrupoCA e GrupoRE.

Tabela 3 - Comparação de desempenho entre turmas de Coeficiente de Rendimento Elevado e o Grupo de Referência

\begin{tabular}{|c|c|c|c|c|c|c|}
\hline & \multicolumn{3}{|c|}{ GrupoRE } & \multicolumn{3}{c|}{ GrupoCA } \\
\hline & $\%$ & $\begin{array}{c}\text { MÉDIA DA } \\
\text { TURMA }\end{array}$ & DESV. PADRAO & $\%$ & $\begin{array}{c}\text { MÉDIA DA } \\
\text { TURMA }\end{array}$ & DESV. PADRAO \\
\hline APROVADOS & $43 \%$ & 7,8 & 1,1 & $74 \%$ & 7,8 & 1,2 \\
\hline REPROVADOS & $27 \%$ & 2,2 & 1,2 & $16 \%$ & 2,3 & 1,4 \\
\hline DESISTENTES & $30 \%$ & & & $10 \%$ & & \\
\hline
\end{tabular}

4.3. Dados Relativos à Reunião das Turmas com Coeficiente alto e baixo com abordagem diferenciada e a turma com os coeficientes misturados.

A Tabela 4 compara o impacto geral que a separação de estudantes em fortes e fracos (os fracos com abordagem diferenciada) com um grupo de estudantes que tem 
os fortes e fracos misturados (sem mudança de abordagem pedagógica). Como anteriormente, as provas foram as mesmas nos dois grupos. Observou-se um desempenho levemente melhor no grupo que teve seus estudantes fortes e fracos separado e teve abordagem diferenciada para os mais fracos:

- Uma média de rendimento levemente inferior para o grupo com coeficiente separados ( 7,7 contra 7,8 para o grupo),

- $10 \%$ a mais de aprovados,

- $1 \%$ a mais de reprovados e

- $13 \%$ a menos de desistentes

Tabela 4: Comparação de desempenho e frequências médios do grupo de referência com a grupo reunião dos grupos alto coeficiente (sem mudança de abordagem ) e baixos coeficientes (com mudança de abordagem).

\begin{tabular}{|c|c|c|c|c|c|c|}
\hline & \multicolumn{3}{|c|}{ GrupoCA + GrupoBD } & \multicolumn{3}{c|}{ GrupoRE } \\
\hline & $\%$ & $\begin{array}{c}\text { MÉDIA DA } \\
\text { TURMA }\end{array}$ & DESV. PADRAO & $\%$ & $\begin{array}{c}\text { MÉDIA DA } \\
\text { TURMA }\end{array}$ & DESV. PADRAO \\
\hline APROVADOS & $55 \%$ & 7,7 & 1,2 & $43 \%$ & 7,8 & 1,1 \\
\hline REPROVADOS & $28 \%$ & 2,6 & 1,7 & $27 \%$ & 2,2 & 1,2 \\
\hline DESISTENTES & $17 \%$ & & & $30 \%$ & & \\
\hline
\end{tabular}

4.4. Dados Relativos à Reunião das Turmas com Coeficiente alto e baixo com e sem abordagem diferenciada e a turma com os coeficientes misturados.

A Tabela 5 totaliza e compara os resultados gerais de desempenho, entre todos os grupos envolvidos no experimento.

Observou-se algumas melhoras em alguns índices, a saber:

- A quantidade de aprovados foi maior (47\%) quando a turma foi dividida por coeficiente de rendimento alto e baixo quando comparada com a turma que não teve essa divisão (43\%).

- A quantidade de reprovados das turmas divididas pelo coeficiente de rendimento foi maior (37\%) quando comparada com a turma que não teve essa divisão (28\%).

- A quantidade de desistentes foi menor para as turmas que foram divididas pelo coeficiente de rendimento (16\%) quando comparada com a turma controle $(29 \%)$.

\section{Discussão}

Apesar do número de estudantes envolvidos ser baixo (322 no total) o experimento trouxe luz a alguns pontos que merecem ser aprofundados em estudos futuros. No que se refere aos estudantes que chegam na disciplina de Fundamentos de Programação 2, com baixo coeficiente de rendimento, os dados obtidos sugerem que a adoção de abordagem didática diferenciada é um estratégia que auxilia os estudantes a melhorarem seu desempenho. 


\subsection{Impacto da separação de fortes e fracos juntamente com abordagem didática diferenciada no fluxo dos estudantes no curso}

Separando-se os estudantes com baixo e alto coeficientes de desempenho acadêmico, observou-se que o número de aprovados aumentou de $10 \%$ ao mesmo tempo em que se diminuiu o número de desistentes e que a média do desempenho acadêmico dos reprovados aumentou de 2,2 para 2,6. Isso pode ser um tipo de ação a ser investigado com o intuito de se minimizar a quantidade de estudantes repetentes em certas disciplinas.

\subsection{Considerações sobre o conceito consolidado de que "misturar estudantes com perfis de desempenho acadêmico variados auxilia os mais fracos a superarem as suas dificuldades"}

Foi uma surpresa observar que tanto os estudantes fortes quanto o fracos, saíram-se melhor quando separados (os fracos com abordagem didática diferenciada). A hipótese que se pode fazer, e necessita de estudos para sua verificação é a de que a manutenção de fortes e fracos só tem sentido quando o conhecimento prévio do grupo

Tabela 5 - Resultado total do experimento - Quantidade de Alunos Aprovados, Reprovados e Desistentes

\begin{tabular}{|l|r|r|r|r|}
\hline \multicolumn{2}{|c|}{$\begin{array}{c}\text { (GrupoCA + GrupoBD + } \\
\text { GrupoBN) Valor } \\
\text { Absoluto }\end{array}$} & $\begin{array}{c}\text { (GrupoCA+GrupoBD + } \\
\text { GrupoBN) \% }\end{array}$ & $\begin{array}{c}\text { (GrupoRE) } \\
\text { Valor } \\
\text { Absoluto }\end{array}$ & \begin{tabular}{c} 
GrupoRE\% \\
\hline APROVADOS
\end{tabular} \\
\hline REPROVADOS & 99 & $47 \%$ & 48 & $43 \%$ \\
\hline DESISTENTES & 77 & $37 \%$ & 31 & $28 \%$ \\
\hline $\begin{array}{l}\text { TOTAL DE } \\
\text { ALUNOS }\end{array}$ & 210 & $16 \%$ & 33 & $29 \%$ \\
\hline
\end{tabular}

de estudantes caracteriza uma curva normal de desempenho. No caso estudado, devido ao processo de entrada na universidade dividir os ingressantes em 5 grupos de cotas (regulamentado por lei federal), a distribuição normal de desempenho dos estudantes que compõem uma turma de entrada deixa de existir. Justificando que a abordagem didática diferenciada apresente melhor resultado do que um tratamento didático igualitário para todos os estudantes.

\subsection{Considerações sobre a natureza da abordagem didática diferenciada.}

Neste experimento, a única mudança realizada foi a solicitação de resolução de exercícios em casa, sua correção e devolução e a posterior socialização de exemplos de como poderiam ter sido resolvidos. Os pesquisadores tem consciência de que a abordagem didática pode ir muito além de designação de tarefas, passando por materiais didáticos específicos, adoção de gêneros de discurso mais adequados, atitudes motivadoras, desenvolvimento de autonomia e autoestima junto aos estudantes. 


\subsection{Considerações sobre o domínio dos pré-requisitos para a disciplina}

Neste experimento não se procedeu a um teste de nivelamento inicial que pudesse identificar a distribuição do nível de competência dos estudantes no que se referia a programação estruturada (tema da disciplina precedente). Este ponto é importante porque a distribuição das notas mostram duas concentrações de notas, uma na parte baixa da escala de desempenho e outra na parte altas, ficando o centro da escala com poucos indivíduos.

\section{Conclusão}

Aumentar os índices de alunos aprovados em qualquer disciplina de um curso superior é um desejo de muitos gestores educacionais (professores, coordenadores de curso, entre outros). Alguns argumentam que esse aumento de índice estaria associado a uma possível piora no no nível de exigência que os professores empregam ou que estaria associado com um conceito de "facilitar" para o aluno passar.

Nessa pesquisa, relata um pequeno aumento no desempenho dos alunos quando turmas são formadas pelo critério de seleção por coeficiente de rendimento acadêmico quando comparada com a turma onde isso não ocorreu. No cômputo geral, houve uma melhora no índice de aprovados (47\% para o grupo que recebeu a intervenção e $43 \%$ para o grupo que não recebeu a intervenção), uma redução nos índices de desistentes (16\% para o grupo que recebeu a intervenção e $29 \%$ para o grupo que não recebeu a intervenção) e um aumento na quantidade de reprovados para o grupo que recebeu a intervenção.

Um aspecto precisa ser destacado ao analisarmos esses números: o grupo que não recebeu a intervenção era formado basicamente por alunos que já tinham cursado a disciplina sem conseguir a aprovação pelo menos uma vez. Se o grupo controle fosse formado por alunos que ainda não cursaram a disciplina, como foi o caso do grupo que recebeu a intervenção, esses números poderiam ser mais promissores.

A redução do índice de desistência vem confirmar os resultados da pesquisa de [Barker et al 2009] que relata que as chances do aluno permanecer no curso aumentam quando ele se sente parte de uma comunidade de mesmo nível social e intelectual. Nesse caso, a turma de alunos onde a intervenção não ocorreu, o GrupoRE, apresentou índice de 30\% de desistentes. Um valor bem superior aos índices apresentados pelas turmas onde ocorreu a intervenção ( índice de $10 \%$ para a turma GrupoCA, 17\% para turma GrupoBN e 28\% para a turma GrupoBD).

O trabalho futuro a ser desenvolvido é investigar quais técnicas ou abordagens pedagógicas poderiam ser empregadas para a turma dos alunos com coeficiente de rendimento baixo de tal forma a melhorar ainda mais esses índices obtidos até o presente momento. Uma dessas técnicas candidatas é a Aprendizagem Baseada em Problemas. Um grupo de estudo foi criado com o objetivo de se capacitar ao uso dessa técnica em sala de aula.

\section{Referências}


Barker, L., J., McDowell, C., Kalahar, K. (2009). Exploring Factors that Influence Computer Science Introductory Course Students to Persist in the Major. ACM SIGCSE Bulletin, 41(2), pp. 282-286.

Citadin, J., R., Kemczinski, A., Matos, A., V. (2014). Formação de Grupos para Aprendizagem Colaborativa: Um mapeamento sistemático da literatura. Nuevas Ideas en Informática Educativa TISE 2014.

Costa, S., S., Cazella, S., Rigo, S.J. (2014). Mineração de dados sobre o desempenho de alunos de cursos de educação permanente em modalidade EAD: Um estudo de caso sobre a evasão escolar na UNA-SUS. CINTED - Novas Tecnologias na Educação, V. 12, No.2, dezembro de 2014.

Gaioso, Natalicia Pacheco de Lacerda (2006) O Fenômeno da Evasão Escolar na Educação Superior no Brasil. Unesco, 2006. Disponível em: www.iesalc.unesco.org.ve/programas/Deserción/Informe. Acesso em $19 / 03 / 2016$

Jesus, S. (2008). Estratégias para motivar os alunos. Revista Educação, 31 (1), 21-29.

Santos, A., Gorgônio, A., Lucena, A., Gorgônio F. (2015). A Importância do Fator Motivacional no Processo Ensino-Aprendizagem de Algoritmos e Lógica de Programação para Alunos Repetentes. Anais do Workshop de Educação em Informática - WEI 2015. XXXV Congresso da Sociedade Brasileira de Computação. 20 a 23 de julho de 2015 - Recife - PE.

Souza, O., S. Morais, P. S., Silva Júnior, F., C. (2015). Um Estudo sobre a Evasão no Curso de Licenciatura em Informática do IFRN - Campus Natal - Zona Norte. Anais do Workshop de Educação em Informática - WEI 2015. XXXV Congresso da Sociedade Brasileira de Computação. 20 a 23 de julho de 2015 Recife - PE.

Manukyan, N., Eppstein, M. J., e Horbar, J. D. (2013). Team structure and quality improvement in collaborative environments. In Collaboration Technologies and Systems (CTS), 2013 International Conference on , IEEE, pp. 523-529.

Manhães, L., M., B., Cruz, S., M., S., Costa, R., J., M., Zavaleta, J., Zimbrão, G. (2012). Identificação dos Fatores que Influenciam na Evasão em Cursos de Graduação Através de Sistemas Baseados em Mineração de Dados; Uma Abordagem Quantitativa. VIII Simpósio Brasileiro de Sistemas de Informação.

Oliveira Junior, J., G., Noronha, R., V., Kaestner, C., A., A., (2014) Análise da Correlação da Evasão de Cursos de Graduação com o Empréstimo de Livros em Bibliotecas. Anais do Congresso Brasileiro de Informática na Educação. Workshop de Mineração de Dados em Ambientes Virtuais do Ensino e Aprendizagem, pag. 601-610. Dourados - Mato Grosso do Sul, 03 a 06 de novembro de 2014. 